\title{
Effects of maternal deprivation on later behavior in two inbred strains of mice
}

\author{
TERRY G. NEWELL \\ BOWLING GREEN STATE UNIVERSITY
}

Mice from two inbred strains were deprived of maternal care for $12 \mathrm{~h}$ per day for $0,4,8$, or 12 days during infancy. The relationship between emotional reactivity and amount of deprivation was described by a $U$-shaped curve for the $B A L B / c$ strain and by an inverted $U$-shaped curve for the $J K$ strain. In avoidance learning, deprived $S s$ from the $J K$ strain performed better than the non-deprived Ss. These results were not in accordance with predictions derived from theories dealing with the effects of infantile stimulation.

Two related areas of psychology provide hypotheses for this experiment. Clinical and child development psychology usually consider the effects of maternal deprivation to be maladaptive and deleterious. Extrapolation to the present study invites the directional predictions that maternally deprived animals will display greater emotional reactivity in an open field test, and show poorer performance in an avoidance learning task than nondeprived controls.

Hypotheses regarding the distributional form of the data come from a theory of infantile stimulation formulated by Denenberg (1964), who proposes that the amount of stimulus input in infancy varies inversely with emotional reactivity in adulthood. In the present study, the nondeprived control animals will be considered the most stimulated group and the maternally deprived animals as the least stimulated. It is thus predicted that a direct relationship will exist between amount of maternal deprivation and emotional reactivity. Denenberg further hypothesizes that an inverted U-shaped curve describes the relationship between amount of infantile stimulation and adult performance in situations such as avoidance learning.

\section{Method}

As each litter from the $B A L B / C$ and $J K$ inbred strains of mice was born it was culled to the size of five and assigned to a treatment group. Starting on Day 4 after birth, the mother was removed from her litter for a period of $12 \mathrm{~h}$ on 4,8 , or 12 consecutive days. The control (0-day) and experimental groups were not otherwise disturbed between birth and weaning (22 days), at which time each $S$ was weighed and individually housed. A litter was included in the study if on the last day of treatment all Ss were still alive. The initial subject pool consisted of $20 \mathrm{Ss}$ for all straintreatment groups except the 12-day JK group which had 25.

From age 30-34 days, each $S$ was removed from his home cage and placed in the center of a brightly lighted 12 gallon wash tub. After $2 \mathrm{~min}$, the $\mathrm{S}$ was removed and the number of boli defecated was recorded. Total amount of defecation over the five day period was used as the measure of emotional reactivity.

At age 60 days Ss were weighed, and between 60-70 days all but 20 BALBs were tested in an avoldance learning situation. Five BALBs from each treatment were tested at age 90 days, but no effect due to age at testing was noted. The test apparatus, a box 24 in. high with an 8 in. sq.grid floor, is fully described in Winston et al (1967). The procedure consisted of placing the $S$ in the center of the grid floor and electrifying the floor after $30 \mathrm{sec}$ had elapsed. An avoidance was defined as preshock emergence from the box through one of three small holes drilled $1 / 2$ in. above the grid. If no escape occurred after $90 \mathrm{sec}$ of shock the trial was terminated. Four trials per day spaced at $30 \mathrm{~min}$ intervals were given for five consecutive days. The measure of learning was the number of avoidance responses made during the 20 trials.

Results

A sex by strain by treatment unweighted means ANOVA was made on each dependent variable. For weight data gathered at weaning and at 60 days, strain has a significant effect $(p<.01)$ at both ages (BALBs heavier than $\mathrm{JK} s)$, while treatment has an effect $(p<.01)$ at weaning but not at 60 days. Sex has an effect $(p<.01$, males heavier then females) at 60 days but not at weaning. All interactions were nonsignificant. An increase in treatment length generally produced a decrease in weight. The correlation between weight at weaning and emotional reactivity was $.33 \pm .11$ for the BALBs and $-.23 \pm .11$ for the JKs; the correlation between weight at 60 days and avoidance learning was $-.18 \pm .12$ for the BALBs and $-.20 \pm .12$ for the $\mathrm{JKs}$. Thus, one concomitant of the maternal deprivation procedure was a nutritional deprivation which influenced emotional reactivity.

The ANOVA for emotional reactivity indicated that strain had a significant effect $(F=18.00$, df $=1 / 139$, $\mathrm{p}<.01)$. Treatment and sex had no overall effect, but there was a treatment by strain interaction ( $F=2.80$, df $=3 / 139, p<.05)$. The nature of the interaction is illustrated in Fig. 1, where the means and sample sizes of each treatment-strain combination are given. The trend of the means for the JK strain is consistent with the hypothesis that maternal deprivation increases emotional reactivity since all three treatment groups are more reactive than the control group. The relationship, however, is not monotonic as predicted by Denenberg's theory, but rather, an inverted U-shaped curve is apparent. Findings for the BALBs are just the 


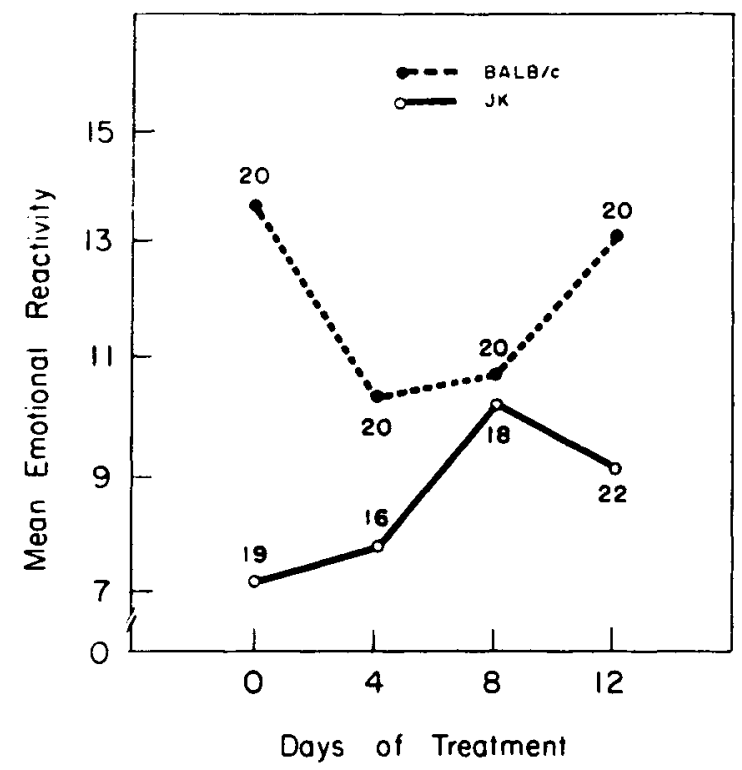

Fig. 1. Emotional reactivity as a function of number of days of maternal deprivation for two inbred strains of mice.

opposite; the treatment groups are all less reactive than the control group, and there is the suggestion of a U-shaped curve. The interaction between genotype and matermal deprivation is especially interesting, for it serves to lllustrate the manner in which behavior can be determined dually by both heredity and environment.

The ANOVA for number of avoidance responses found strain to be the only significant effect ( $F=27.09$, df $=1 / 134, p<.01$ ). However, a sex by treatment ANOVA performed on each strain indicated that treatment was significant $(F=3.42, d f=3 / 63, p<.05)$ in the JK strain. Figure 2 gives the means and sample sizes for each treatment-strain group; the hypothesized inverted Ushaped curves are not present.

The directional hypothesis that a general performance decrement would be found was also not supported. The results indicate, in fact, that in the JK strain treated Ss are superior to control $\mathrm{Ss}$ in avoidance learning. Discussion

None of the hypotheses generated by a consideration of the research on maternal deprivation in children or by Denenberg's theory of the effects of infantile stimulation were conclusively supported. The maternal deprivation treatment had no consistent effect on emotional reactivity. The trend of the data for the JK strain

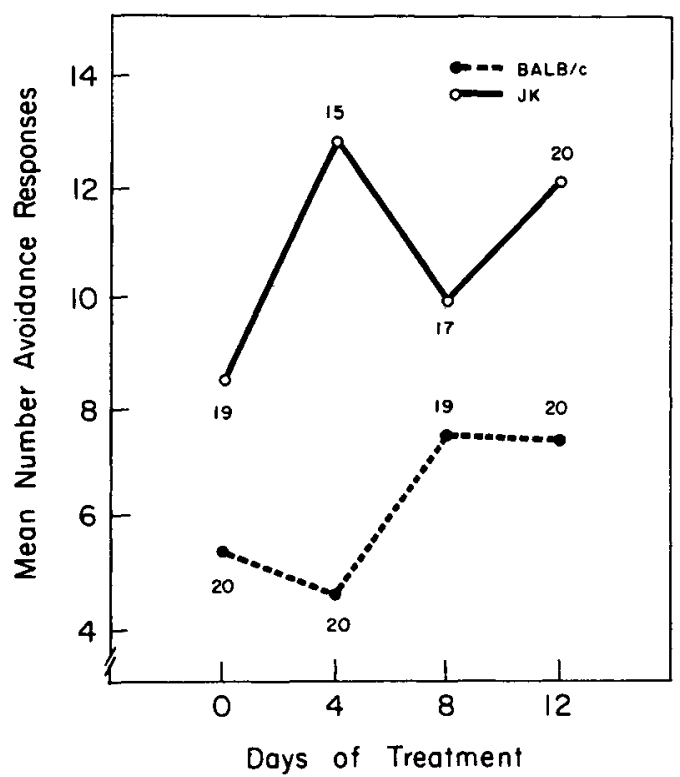

Fig. 2. Avoidance learning as a function of number of days of maternal deprivation for two inbred strains of mice.

supports the prediction that emotional reactivity is greater in deprived Ss, but an opposite result was obtained for the BALB strain. The results of the avoidance learning test do not support the prediction that deprived animals are poorer learners; rather, the deprived Ss in the JK strain are better learners. Denenberg's theory predicts that a monotonic relationship exists between amount of deprivation and emotional reactivity and that a U-shaped curve describes the relationship between amount of deprivation and avoidance learning. Neither of these predictions was in evidence. It appears that the maternal deprivation procedure employed here does not act to determine the effects of infantile experience on later behavior in the manner Denenberg proposes.

\section{References}

DENENBERG, V. H. Critical periods, stimulus input, and emotional reactivity: A theory of infantile stimulation. Psychol Rev., 1964, 71, 335-351.

WINSTON, H., LINDZEY, G., \& CONNER, J. Albinism and avoidance learning in mice. J. comp. physiol Psychol, 1967, 63, 77-81.

\section{Note}

1. The author was supported by a NSF Summer Fellowship while conducting this research. Preparation of the report was aided by USPHS Grant MH 6964 to the Center for Personality Research, University of Minnesota. 